\title{
Idiopathic Lenticulostriate Artery Aneurysm in an Adolescent Presenting with Intracerebral Hemorrhage
}

Che-Kuang Lin, Yi-Hsin Tsai, Shih-Hao Huang and Lin-Hsue Yang*

Division of Neurosurgery, Department of Surgery, Far Eastern Memorial Hospital, Banqiao, New Taipei, 22060, Taiwan

\begin{abstract}
We describe a rare case of idiopathic lenticulostriate artery (LSA) aneurysm in a 15-year-old girl who presented in the emergency department with a sudden onset of severe headache and left hemiparesis. Computed tomography revealed an intracerebral hemorrhage in right basal ganglion. Angiography revealed a LSA aneurysm. The patient underwent right frontal craniotomy for aneurysm removal. Overall, the patient experienced a positive outcome after surgery for a ruptured LSA aneurysm. Following the operation, the patient participated in a 1-week rehabilitation program, which improved her left hemiparesis. We observed a positive outcome with surgical treatment for the ruptured LSA aneurysm, which was probably improved by early surgical intervention.
\end{abstract}

Keywords: Aneurysm; Basal ganglia; Intracranial hemorrhage; Lenticulostriate vasculopathy

\section{Introduction}

Lenticulostriate artery (LSA) aneurysm is a rare disease that has a low incidence in adolescents. Even for adults and children, there are few cases of LSA aneurysm reported in the literature [1]. Here, we describe a distal LSA aneurysm in a 15 -year-old girl that presented with intracerebral hemorrhage (ICH) in the basal ganglia. Our report describes that the diagnosis, management, and outcome in this case. We believed that this case report is the first to document an LSA aneurysm an aneurysm in adolescence, which included surgical intervention with positive outcomes.

\section{Case Report}

\section{History and examination}

A 15-year-old girl presented in our emergency department with a sudden onset of headache and left-sided weakness that begun while playing video games. Vomiting and sleepiness, without loss of consciousness, followed the initial symptom onset. She was previously healthy and without a history of perinatal insults, recent infection or neurological symptoms. On admission, she was alert but showed leftsided hemiparesis with difficulty moving against the gravity. Computed tomography showed right putaminal ICH (Figure 1) with mass effect.

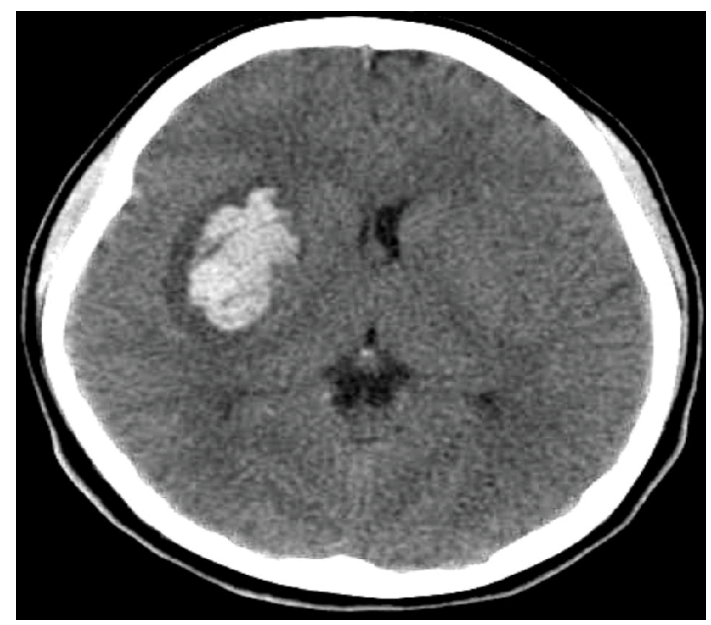

Figure 1: Axial CT scan indicating hemorrhage in the right putamen and obvious mass effect.
Diagnostic angiography revealed an aneurysm in the distal portion of the LSA (Figure 2). After the image study, the patient appeared drowsy, with decreased consciousness, and demonstrated an obvious mass effect from the ICH, we therefore decided to surgically remove the aneurysm. We performed a right frontal craniotomy and a small corticotomy that provided the shortest distance between the cortical surface and the hematoma. Consistent with the angiography, the aneurysm was identified at the hematoma site, which was divided at its proximal end and excised with direct cauterization (Figures 3 and 4).

Postoperative course: The patient experienced an uneventful postoperative recovery and was transferred for rehabilitation. During
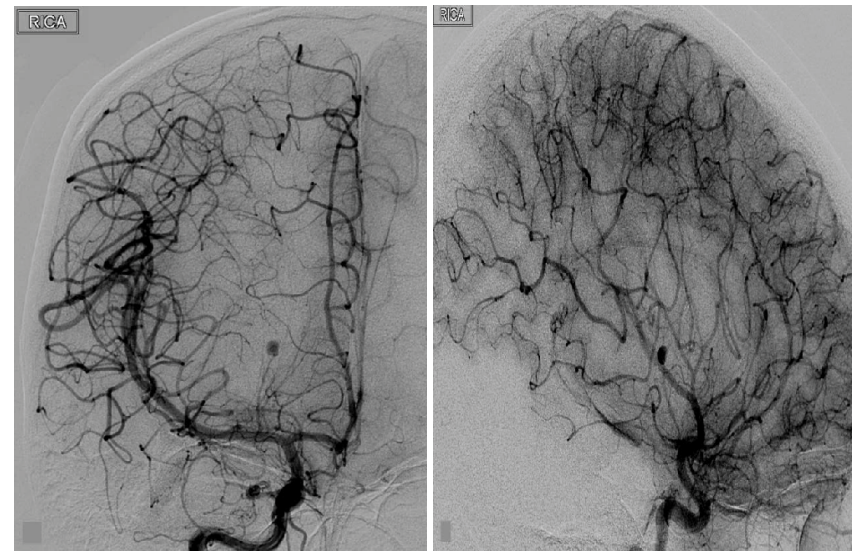

Figure 2: Right carotid angiogram. Anteroposterior and lateral views demonstrate the aneurysm (white arrow) arising from the distal lenticulostriate artery.

*Corresponding author: Dr. Lin-Hsue Yang, M.D., Division of Neurosurgery, Department of Surgery, Far Eastern Memorial Hospital ,21 Nan-Ya South Road, Section2, Banqiao, New Taipei, 22060, Taiwan, Tel: +886-2-89667000 +886919225010; Fax: +886-2-2935-6940; E-mail: tetsukohayashi@hotmail.com

Received August 18, 2015; Accepted November 12, 2015; Published January 08, 2016

Citation: Lin CK, Tsai YH, Huang SH, Yang LH (2016) Idiopathic Lenticulostriate Artery Aneurysm in an Adolescent Presenting with Intracerebral Hemorrhage. Surgery Curr Res 6: 256. doi:10.4172/2161-1076.1000256

Copyright: (c) 2016 Lin CK, et al. This is an open-access article distributed under the terms of the Creative Commons Attribution License, which permits unrestricted use, distribution, and reproduction in any medium, provided the original author and source are credited. 
Citation: Lin CK, Tsai YH, Huang SH, Yang LH (2016) Idiopathic Lenticulostriate Artery Aneurysm in an Adolescent Presenting with Intracerebral Hemorrhage. Surgery Curr Res 6: 256. doi:10.4172/2161-1076.1000256

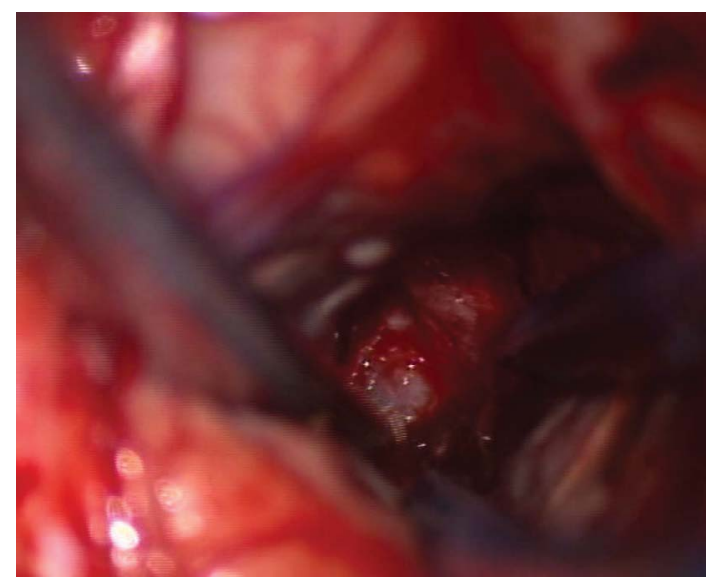

Figure 3: Intraoperative image for LSA aneurysm.

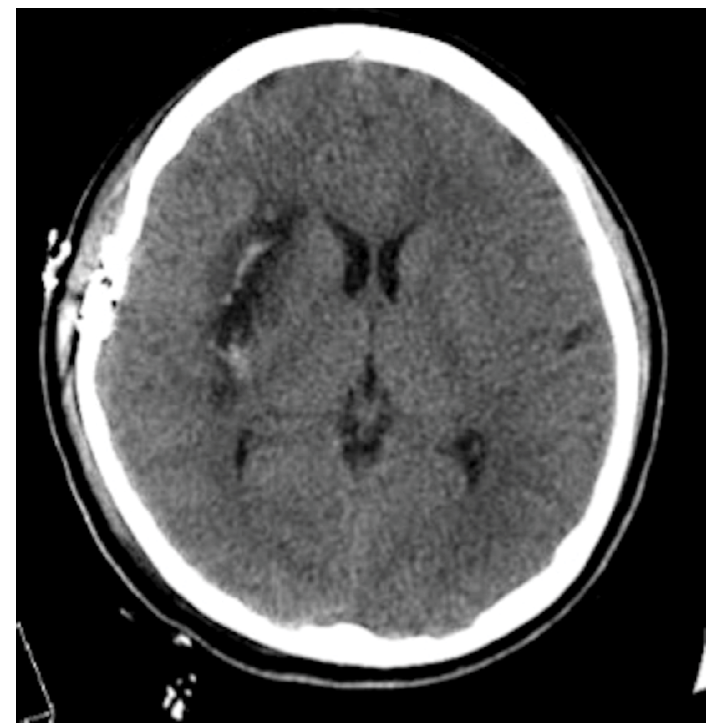

Figure 4: Immediate postoperative computed tomography. It revealed little residual hematoma without mass effect.

her hospitalization, cardiac echo showed no valvular vegetation, and a hemogram failed to reveal signs of infection. Rheumatoid factor, antinuclear antibody, and erythrocyte sedimentation rate were checked; all of which were negative. Her left hemiparesis improved with rehabilitation, and she walked after 1 month with limited assistance. Microscopic examination of the vasculature revealed focal cleft formation and hemorrhagic clots, which are compatible with aneurysm (Figure 5).

\section{Discussion}

ICH in childhood and adolescence is rare and most often results from arteriovenous malformation, hematologic abnormality, or brain tumor [1,2]. Other etiologies include intracranial aneurysm, cavernous hemangioma, vasculopathy, vasculitis, cerebral and systemic infections, and, rarely, illicit drug use [1,2]

Intracranial aneurysms are the most frequent cause of $\mathrm{ICH}$, but they rarely occur in childhood and adolescence [1, 2]. Furthermore, the location of the aneurysm in this particular case, which was in the distal portion of LSA, was unusual. A literature review revealed a total of 19 cases reported in adults, and only four reported in children [1-5]. All four childhood cases presented with both ganglionic hemorrhage and intraventricular hemorrhage [1-5]. Our case, however, is the first to show an LSA aneurysm in adolescence (Table 1).

Most LSA aneurysms are idiopathic, and are caused by Moyamoya disease, rheumatic diseases, arteriovenous malformation, and intraventricular tumor [1-5]. In Moyamoya disease and brain tumors that produce a feeding artery with aneurysm, hemorrhaging can occur due to a pseudoaneurysm or dilated vessel, which compensates for the occlusive change in the main trunk of the cerebral perfusion [3-5] Vasculitis associated with distal cerebral aneurysms is rarely reported in the literature [6]. Infectious intracranial aneurysms more often affect children, particularly those with congenital heart disease, artificial heart valves, rheumatic heart disease, or acquired bacterial endocarditis [7]. Nevertheless, no case of infectious LSA aneurysm has been reported [1]. In our case, cardiac echo failed to reveal the vegetation at the valve, and rheumatoid disease was not indicated.

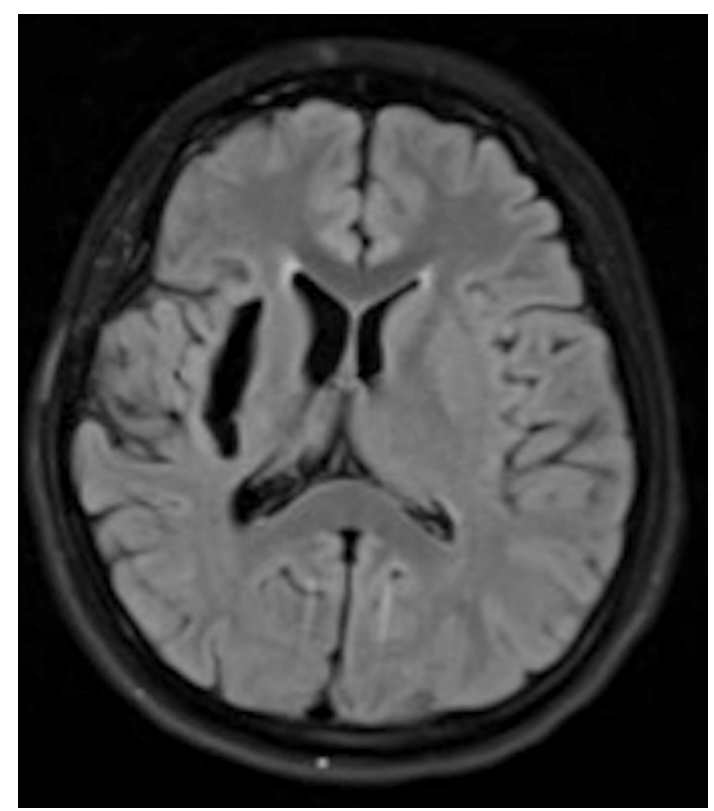

Figure 5: Postoperative image one year after the operation. Magnetic resonance image (MRI) showed right deep brain parenchymal resorption and encephalomalacia change.

\begin{tabular}{|c|c|c|c|c|c|c|c|}
\hline Authors \& Year (Reference No.) & Age, Sex & History & CT findings & Possible cause & Treatment & Pathology & Outcome \\
\hline Endo et al., 1996 [3] & $12 \mathrm{yrs}, \mathrm{F}$ & NR & $\mathrm{ICH}$ and IVH & Idiopathic & Excision & Not reported & Good \\
\hline Kaptain et al., 2001 [4] & $2 \mathrm{mos}, \mathrm{M}$ & Lethargy and vomiting & $\mathrm{ICH}$ and IVH & Idiopathic & Excision & True aneurysm & Good \\
\hline Lehmann et al., 2003 [5] & $2 \mathrm{yrs}, \mathrm{F}$ & Conscious change and meningeal sign & $\mathrm{ICH}$ and IVH & Idiopathic & Excision & True aneurysm & NR \\
\hline Matushita et al., 2007 [1] & 5 yrs, F & Headache and vomiting & $\mathrm{ICH}$ and IVH & Idiopathic & Excision & True aneurysm & Good \\
\hline Present study & 15 yrs, F & Headache and left hemiparesis & $\mathrm{ICH}$ & Idiopathic & Excision & True aneurysm & Good \\
\hline
\end{tabular}

$\pi / \mathrm{CH}=$ intracerebral hemorrhage; IVH = intraventricualr hemorrhage; NR = not reported.

Table 1: Literature review of the reported cases of ruptured distal LSA aneurysm in childhood and adolescence ${ }^{\pi}$ 
Citation: Lin CK, Tsai YH, Huang SH, Yang LH (2016) Idiopathic Lenticulostriate Artery Aneurysm in an Adolescent Presenting with Intracerebral Hemorrhage. Surgery Curr Res 6: 256. doi:10.4172/2161-1076.1000256

Page 3 of 3

The rare distal LSA aneurysm is located deep inside the basal ganglia. On rupture, it patients will present with ICH $[1,4,5]$. ICH patients receiving a conventional craniotomy often have poor survival (22\%-36\%) and functional outcome (44\%-74\%) [8]. The incidence of rebleeding in LSA aneurysm, however, is unknown due to its rare occurrence. Previous case reports describe conservative treatment in ruptured LSA aneurysm that resulted in either the spontaneous thrombosis and obliteration of the aneurysm [9], or rebleeding resulting in death [10]. Gandhi et al. [11] described positive outcomes following the surgical treatment of ruptured idiopathic LSA aneurysm [1]. In our case, we noted neurological deterioration. The LSA aneurysm formed a hematoma upon rupture, which indicated tis location on threedimensional angiography and provided operative space that required less retraction [11]. Considering intracranial hematoma contributes to the local mass effect, elevates intracranial pressure, and elicits pathological cascades that produce biochemical toxicity, both early and complete removal of ICH can considerably reduce the secondary injury caused by ICH [12]. In our case, the hematoma was readily removed with suction, which mediated early surgical treatment within 12 hours [8]. Another concern is the preserving the parent vessel during aneurysm extirpation. According to cases reported in the literature, sacrificing the parent LSA has been well tolerated despite the radiographic changes [11]. In the rare case of idiopathic LSA aneurysm, the rebleeding rate is unknown and spontaneous obliteration is unlikely. Considering the risk of secondary injury resulting from ICH and the possibility of rebleeding, we further conclude that confirmed LSA aneurysms should be treated with early surgical intervention.

\section{Conclusion}

LSA aneurysm is rare, and the present report is the first to describe LSA aneurysm in adolescence. We observed a positive outcome with surgical treatment for a ruptured LSA aneurysm, and that early surgical treatment probably improved this outcome with suctionable ICH.

\section{References}

1. Matushita H, Amorim RLO, Paiva WS, Cardeal DD, Pinto FC (2007) Idiopathic distal lenticulostriate artery aneurysm in a child. Journal of Neurosurgery: Pediatrics 107: 419-424.

2. Jordan LC, Hillis AE (2007) Hemorrhagic stroke in children. Pediatr Neurol 36 : 73-80.

3. Endo M, Ochiai C, Watanabe K, Yoshimoto Y, Wakai S (1996) Ruptured peripheral lenticulostriate artery aneurysm in a child: case report. No Shinkei Geka 24: 961-964.

4. 4. Kaptain GJ, Sheehan JP, Kassell NF (2001) Lenticulostriate aneurysm of infancy. J Neurosurg 94: 538-538.

5. Lehmann P, Toussaint P, Depriester C, Legars D, Deramond H (2003) Lenticulostriate aneurysms. Radioclinical study. J Neuroradiol 30: 115-120.

6. Nakasu S, Kaneko M, Matsuda M (2001) Cerebral aneurysms associated with Behcet's disease: a case report. Journal of Neurology, Neurosurgery \& Psychiatry 70: 682-684.

7. Bingham WF (1977) Treatment of mycotic intracranial aneurysms. J Neurosurg 46: $428-437$.

8. Kuo L-T, Chen C-M, Li C-H, Tsai J-C, Chiu H-C, et al. (2011) Early endoscopeassisted hematoma evacuation in patients with supratentorial intracerebral hemorrhage: case selection, surgical technique, and long-term results. Neurosurg Focus 30: E9.

9. Gupta AK, Rao VR, Mandalam KR, Kumar S, Joseph S, et al. (1989) Thrombosis of multiple aneurysms of a lateral lenticulostriate artery. An angiographic followup. Neuroradiology 31: 193-195.

10. Kidoguchi J, Chiba M, Murakami T, Saiki I, Kanaya H, et al.(1987) [A case of systemic lupus erythematosus associated with an aneurysm of the lenticulostriate artery]. No Shinkei Geka 15: 1221-1225.

11. Gandhi CD, Gilad R, Patel AB, Haridas A, Bederson JB (2008) Treatment of ruptured lenticulostriate artery aneurysms. J Neurosurg 109: 28-37.

12. Zuo Y, Cheng G, Gao D-K, Zhang X, Zhen HN, et al. (2009) Gross-total hematoma removal of hypertensive basal ganglia hemorrhages: A long-term follow-up. Journal of the neurological sciences 287: 100-104. 